International Journal of Pure and Applied Mathematics

Volume 114 No. 4 2017, 819-822

ISSN: 1311-8080 (printed version); ISSN: 1314-3395 (on-line version)

url: http://www.ijpam.eu

doi: 10.12732/ijpam.v114i4.11

\title{
GENERALIZED LITTLE'S FORMULAS AND CLASSIFICATION OF HIGHER ORDERS QUEUES IN THE QUEUING THEORY
}

\author{
Alexander Kirpichnikov ${ }^{1}$, Anton Titovtsev ${ }^{2} \S$ \\ ${ }^{1,2}$ Kazan National Research Technological University \\ 68, K. Marksa, Kazan, 420015, RUSSIA
}

\begin{abstract}
The paper provides generalized Little's formulas for the N-th order queues in queuing systems of various types.
\end{abstract}

AMS Subject Classification: $60 \mathrm{~K} 25$

Key Words: queue, quality of service (QoS), queuing system, service facility

\section{Introduction}

As it is known, mean processing time of one claim in the system $\bar{t}_{S}$, mean staying time of claims in the queue $\bar{t}_{W}$ and the common mean staying time of the claim in the system in general $\bar{t}_{T}=\bar{t}_{S}+\bar{t}_{W}$ for Markov queuing systems (QS) are bound to corresponding discrete characteristics of QS by three following formulas [1], [2]:

$$
\bar{t}_{S}=\bar{n} / A ; \quad \bar{t}_{W}=\bar{l} / A ; \quad \bar{t}_{T}=\bar{k} / A,
$$

where $A$ is throughout capacity of the system, i.e. anaverage number of claims served by the system in unity of time. Discrete characteristics of the system are understood respectively as an average number of busy channels $\bar{n}$, mean

Received: January 27, 2017

Revised: $\quad$ April 7, 2017

Published:

June 7, 2017 (c) 2017 Academic Publications, Ltd. url: www.acadpubl.eu

$\S_{\text {Correspondence author }}$ 
length of the queue $\bar{l}$, and an average number of claims in the system in general $\bar{k}=\bar{n}+\bar{l}$. Sometimes, these formulas are written in the form

$$
\bar{t}_{S}=\bar{n} / \lambda ; \quad \bar{t}_{W}=\bar{l} / \lambda ; \quad \bar{t}_{T}=\bar{k} / \lambda,
$$

when the total intensity of claims stream $\lambda$ coming into the system is in the denominator.

In fact, however, the denominator of these formulas should not be made of the total intensity of claims stream but only of that part which corresponds to those claims that are really transferred through the system (more precisely, through the service facility), i.e. absolute throughout capacity of the system $A$.

Formulas (1) are commonly called Little's formulas. At first, the result which engineers used for a long time, existed as several empirical formulas, i.e. in the form of some kind of "folkloric theorem", as they say. Apparently, J. D. C. Little was the first person who gave it a strict formulation in 1961. The intuitive proof of Little's formulas comes to the fact that in a steady state the next demand entering the system finds in it the same average number of demands which remains in the system when this demand leaves it. This value is just equal to the product of claims stream intensity transferred through the system (or its any subsystem) multiplied by the mean time of their staying in this system (subsystem):

$$
\bar{n}=A \bar{t}_{S} ; \quad \bar{l}=A \bar{t}_{W} ; \quad \bar{k}=A \bar{t}_{T} .
$$

Direct mechanical analog of formulas (2) is a well-known relation for the way passed at a steady movementsbased on moving velocity $v$ and travel time $t$.

$$
s=v t .
$$

The case is different with QS numerical characteristics concerning higher orders queues in these systems. Let us remind that we have called the $\mathrm{N}$-th order queue as the queue that is calculated as long as there is a minimum of $\mathrm{m}+\mathrm{N}$ claims in the system; $N$ of them is in the memory [4]. Such queue exists if a newly arrived claim finds a minimum claims $\mathrm{N}$ waiting for service before it.

When $\mathrm{N}=0$ we have a physical queue which is studied in work [3] in detail. In the GPSS World simulation modeling system this characteristic has the name "queue without zero inputs". When $\mathrm{N}=1$ we have the so-called real queue [1], [2]; in case $\mathrm{N}>1$ we have higher orders queues.

As it is shown in the work [3], corresponding relations have quite a similar form (1) for a physical queue

$$
\overline{\tilde{t}_{W 0}}=\overline{\tilde{l}_{0}} / A
$$


however this formula becomes unjust for higher orders queues.

It was found out in works [1], [2] and [4], that for systems of $\mathrm{M} / \mathrm{M} / \mathrm{m}$ and $\mathrm{M} / \mathrm{M} / \mathrm{m} / \mathrm{E}$ classes (though, all numerical characteristics of the first ones can be obtained by a limit transference from numerical characteristics of the second ones) the following relation is performed

$$
\overline{\tilde{t}_{W 1}}=\overline{\tilde{l}_{1}} / m \mu
$$

where $m$ is the number of channels in the serving multi-channel facility, and $\mu$ claims service intensity by one channel, i.e. areal queue moves with $m \mu$ velocity. It is possible to show that the same dependence will remain true for all types of higher orders queues for which $\mathrm{N}>1$.

$$
\overline{\tilde{t}_{W N}}=\overline{\tilde{l}_{N}} / m \mu
$$

Relations (1)-(5) connect parameters of usual mathematical, physical and real queues in open queuing systems and parameters of higher orders queues in these systems as well. It is clear that these relations will be absolutely similar for close-loop queuing systems. At the same time it is possible to call the obtained system of formulas (1)-(5) as generalized Little's formulas.

As we see, all higher orders queues in queueing systems of various types from the point of view of claims traveling velocity in these queues can be divided into two unequal classes. Only two types of queues will belong to the first class: they are usual mathematical and physical queues which move with a transferring velocity of claims through the system $A$. The real queue and all higher orders queues for which according to the definition we have $\mathrm{N}>1$ belong to the second more extensive class. These queues move with the service velocity $m \mu$.

Further, works [1] and [2] provide formulas obtained for the mean length of a real queue for queuing systems of various types. In particular, the expression for a mean length of a real queue of the system with an unlimited memory volume (within G. Kendall's symbolism - M/M/m model) is the following

$$
\overline{\tilde{l}_{1}}=\frac{m}{m-\rho},
$$

where $\rho=\lambda / \mu$ is the given intensity of claims stream entering the system. The similar expression for a physical queue is obtained in work [3]:

$$
\overline{\tilde{l}_{0}}=\frac{\rho}{m-\rho} .
$$


But for the $\mathrm{M} / \mathrm{M} / \mathrm{m}$ model $A=\lambda$, and then according to formulas (3)-(4) we have

$$
\overline{\tilde{t}_{W 0}}=\frac{\overline{\tilde{l}_{0}}}{\lambda}=\frac{1}{\mu(m-\rho)} ; \quad \overline{\tilde{t}_{W 1}}=\frac{\overline{\tilde{l}_{1}}}{m \mu}=\frac{1}{\mu(m-\rho)},
$$

i.e. for the model with an unlimited queue the mean staying time of one claim in a physical queue coincides with the mean staying time of the claim in a real queue: $\overline{\tilde{t}_{W 0}}=\overline{\tilde{t}_{W 1}}$. The obtained result can be called the theorem on physical and real queues in queuing systems with an unlimited memory volume.

\section{References}

[1] A.P.Kirpichnikov Prikladnaya teoriya massovogo obsluzhivaniya [Applied queuing theory], Publishing office of KSU Publ., Kazan (2008), In Russian.

[2] A.P.Kirpichnikov Metody prikladnoy teorii massovogo obsluzhivaniya [Methods of applied queuing theory], Publishing office of KSU Publ., Kazan (2011), In Russian.

[3] A. Kirpichnikov, A. Titovtsev, Physical and mathematical queues in the applied queuing theory, International Journal of Pure and Applied Mathematics, 108, No. 2 (2016), 409418.

[4] A. Titovtsev, The concept of higher orders queues in the queuing theory, International Journal of Pure and Applied Mathematics, 109, No. 2 (2016), 451-457. 